Article

\title{
A Performance Analysis of VANETs Propagation Models and Routing Protocols
}

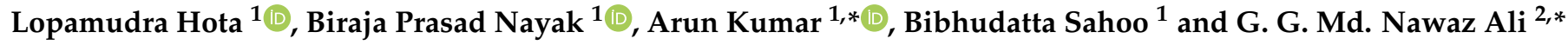 \\ 1 Department of Computer Science \& Engineering, National Institute of Technology, Rourkela 769008, India; \\ 519cs1015@nitrkl.ac.in (L.H.); 519cs1005@nitrkl.ac.in (B.P.N.); bdsahu@nitrkl.ac.in (B.S.) \\ 2 Department of Computer Science and Information Systems, Bradley University, Peoria, IL 61625, USA \\ * Correspondence: kumararun@nitrkl.ac.in (A.K.); nali@fsmail.bradley.edu (G.G.M.N.A.)
}

Citation: Hota, L.; Nayak, B.P.;

Kumar, A.; Sahoo, B.; Ali, G.G.M.N. A

Performance Analysis of VANETs

Propagation Models and Routing

Protocols. Sustainability 2022, 14, 1379.

https://doi.org/10.3390/su14031379

Academic Editor: Mouloud Denai

Received: 23 November 2021

Accepted: 18 January 2022

Published: 26 January 2022

Publisher's Note: MDPI stays neutral with regard to jurisdictional claims in published maps and institutional affiliations.

Copyright: (c) 2022 by the authors. Licensee MDPI, Basel, Switzerland. This article is an open access article distributed under the terms and conditions of the Creative Commons Attribution (CC BY) license (https:// creativecommons.org/licenses/by/ $4.0 /)$.

\begin{abstract}
Vehicular Ad hoc Networks (VANETs) thrive on providing a communication channel between vehicles and infrastructures that facilitate efficient and safe Vehicle-to-Vehicle (V2V) as well as Vehicle-to-Infrastructure (V2I) communications. Reliable and efficient transmission amongst vehicles and Road Side Units (RSUs) is a prime concern of Intelligent Transportation System (ITS). One of the primary challenges involved in vehicular communication is designing an efficient routing mechanism for data dissemination from node to node via a reliable route. The harsh vehicular environment with varied road conditions and obstacles in the signal propagation path induces another challenge. Therefore, this paper presents a comparative analysis of existing routing protocols with propagation models to assist researchers in gaining insight into the existing propagation model and routing protocols. The study also optimizes routing and propagation models for reliable packet dissemination. This work uses a realistic scenario from Open Street Map (OSM), and simulations are performed using SUMO. The trace files generated from SUMO are used for further simulation in NS-3. The simulation results are presented and studied in detail. The results show that the Two-Ray Ground and FRIIS propagation model outperforms the compared models, and the routing protocol OLSR outperforms AODV and DSDV.
\end{abstract}

Keywords: comparative analysis; performance; VANETs; propagation model; routing

\section{Introduction}

There has been remarkable growth in the number of vehicles in the Global Transportation System (GTS) in the last decades. Rapid urbanization has led to an immense expansion in vehicles for transportation, thus increasing research in VANETs. It is a crucial component of Intelligent Vehicular System (IVS) that uses various communication technologies such as short-range WLAN and cellular technologies such as LTE and VoLTE, $\mathrm{WiFi}$ (IEEE $802.11 \mathrm{~b} / \mathrm{g}$ ), Zigbee, etc. VANET topology is dynamic and characterized by fast-moving nodes communicating via V2V, V2I, and V2X modes of communication. Since 1980, VANETs have been growing in their research, where vehicles interact through wireless network communication. The vehicular communication range is around 100-1000 m, and the two media of communications are via On-board Units (OBUs) or Road-Side Units (RSUs) [1]. The OBUs are mounted on the vehicles, whereas RSUs are fixed infrastructure placed on the street or intersections and serve as access points. Vehicles route messages through OBUs and RSUs.

The United States Federal Communication Commission (FCC) has allotted a frequency band of 5.850-5.925 GHz to hold up with vehicular communication, i.e., V2V and V2I. Further, the allocated band of $5.9 \mathrm{GHz}$ consists of seven non-overlapping channels with $10 \mathrm{MHz}$ channel bandwidth of each track. The use of Dedicated Short-Range Communication (DSRC), IEEE 802.11p [2], and Wireless Access for Vehicular Environment (WAVE) (IEEE 1609 protocol stack [3]) has allowed efficient communication within VANETs. These mechanisms provide a high data rate, reliable link establishment, less latency. MAC layer 
implements the CSMA/CA protocols for WAVE standards to adjust to dynamic network topology and increased mobility.

In addition, many traditional routing protocols such as AODV, DSDV, OLSR are implemented and enhanced for efficient route detection to disseminate messages from the source to destination. MMPR-OLSR in [4] is proposed to optimize data dissemination and better channel utilization with a robust VANETs architecture and multipoint selection of relay. Radio propagation models play a vital part in the performance of a network predicting the propagation behaviour.

Figure 1 depicts a V2V and V2I communication scenario with a sender and receiver vehicle where other vehicles, RSUs, and buildings act as reflectors causing path loss. Path loss is computed by considering Line-Of-Sight (LOS) and Non-Line-Of-Sight (NLOS) between vehicles and other units. The difference between LOS and NLOS relates to the cumulative distance between sender and receiver, including intermediate reflectors. Propagation models for the channel in a vehicular environment are related to road traffic density, vehicle speed, and scenarios such as highway or urban.

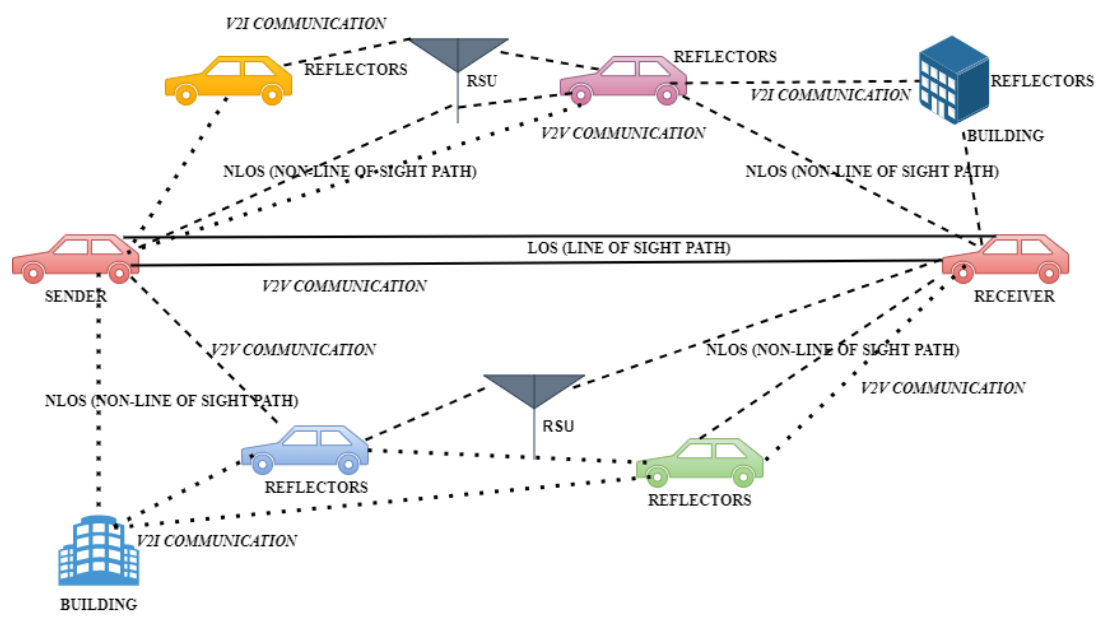

Figure 1. Vehicular (V2V and V2I) communication.

In VANETs, various radio models estimate path loss in different scenarios, including flyovers, buildings, tunnels, and other infrastructures. Based on the above consideration, this work compares topology-based routing protocols with different propagation models and a varied number of nodes (vehicles), changing simulation time to reach the performance levels. Specifically, the simulation was conducted by taking a real-time traffic scenario of Rourkela, India, using SUMO [5] on various existing routing protocols and propagation models. The performance analysis is based on three basic parameters-packet delivery ratio, throughput, and delay. The main contribution of this work includes a generic study of different routing protocols and propagation models for VANETs in a realistic traffic setting. This work analyses the impact of existing propagation models and routing protocols simulated by varying node number (vehicles) and simulation time on network performance.

The primary contribution of the paper includes:

1. A study of implementation and design of an efficient routing mechanism in VANETs;

2. Study and analysis of some basic routing protocols and propagation models on performance evaluation of VANETs;

3. The performance evaluation is based on throughput, packet delivery ratio, delay, goodput, and overhead observed for routing protocols and propagation models. Simulations carried out by taking a realistic scenario from an open street map and performance based on the mentioned metrics are analysed.

The rest of the paper is organized as follows. Section 2 presents the relevant existing studies. Section 3 introduces the proposed work, specifically, the models and protocols used 
in this work. The performance metrics and simulation setup along with results obtained are described and discussed in Section 4. Finally, the paper is concluded in Section 5.

\section{Literature Review}

In a VANET, there may be thousands of vehicles and several network areas, which is in contrast to the MANET, where nodes are restricted to only a few hundred in specific areas. There are unevenly distributed nodes in VANETs. For example, urban areas have dense nodes, while highways are sparse. Due to this, it would be difficult to disseminate information that needs to be time-constrained. Routing protocols should be designed for VANET environments to achieve scalability, efficiency, and comparability. The subsection that follows discusses several routing protocols for VANETs.

Many studies in the past have focused on routing protocol performance analysis in Mobile Ad-hoc Network (MANET) [6], as well as in Flying Ad-hoc Networks (FANET) [7]. Authors in [8] have compared various routing protocols and radio propagation models with real-time data sets. A comparative review of routing protocols implemented for the VANETs scenario is presented in [9] along with challenges and open issues for research directions.

The paper [10] presents a method for disseminating data in MANETs and VANETs via mobile software agents. Agents' decisions about migrating among the network nodes are supported by geographic information about the nodes so that the desired dissemination of data is achieved. Three distinct levels of information completeness are used to compare and contrast intelligent decision levels. The experiments suggest that increased information richness is effective in keeping agents in their respective Target Regions (TR).

Narayan et al. (2020) [11] examined an ideal protocol for VANET using various network metrics in DSDV, AODV, AOMDV, and GPSR topologies. For urban mobility, the nodes were visualized without the road layout using SUMO and NS-2. Based on the results, AODV is preferred for throughput, delay, packet delivery ratio, and AOMDV for the least overhead. For congestion control, DSDV is more likely to be effective using GPSR

Wang et al. (2017) [12] studied using the existing routing protocols of AODV, DSR, and Cluster-Based Routing Protocol (CBRP) for VANETs, specifically at road intersections. Various traffic density settings and the number of vehicle nodes were used to configure the settings. VanetMobiSim and NS-2 were used to simulate the scenario. As a result, AODV, DSR, and CBRP perform equally well at low densities. However, CBRP outperforms compared protocols even in high density with less packet loss.

To reduce packet loss ratio in VANET, Benmir et al. (2019) [13] explored the GPSR routing protocol. This paper proposes a second route for sending the same packet to reduce the ratio. Veins, SUMO, and OMNET++, were used to experiment. As a result of the simulation, the improved GPSR model outperforms the existing GPSR in packet delivery ratio.

The authors in [14] quantified the performance of AODV, AOMDV, and DSDV in the NS-2 simulator for MANET. Results show that AOMDV outperformed other protocols in terms of packet delivery ratio. At the same time, DSDV showed better results in terms of latency. Throughput of AODV is better than the other protocols in a low-density scenario.

Sallum et al. [15] provided analysis of routing protocols for VANETs based on AODV, OLSR, DSDV changing the mobility model (BonnMotion), trajectories, varying speed, and network density. Amina et al. [16] compared OLSR, GPCR, and GSR considering vehicle density. The metrics for performance evaluation were packet delivery ratio, throughput, overhead, and end-to-end delay evaluated in NS-3 using SUMO.

Angeles et al. [17] compared AODV, DSR, DYMO, and OLSR under various propagation models taking a realistic scenario of Brazil and evaluating different metrics by $\mathrm{OMNeT}++$ simulator. Similarly, Shuhaimi et al. [8] implements various propagation models on AODV and computed parameters, including packet loss, throughput, and delay for performance analysis, taking the scenario of Selangor and compared using NS-2 simulator.

Several literature contributions have emphasized the use of MIMO techniques to exploit multipath. In future vehicular networks, massive MIMO with beam-forming is 
being investigated as a potential wireless communication technique. It should be noted that the mixed noise in a complex environment such as a vehicular network is prevalent using MIMO [18].

In contrast to the literature study, this work attempts to analyze the performance of OLSR, AODV, and DSDV protocols while considering propagation models as a constant. The performance of various propagation models is compared, considering the OLSR as a routing protocol. For interfacing MAC and PHY layer, $802.11 \mathrm{p}$ is assumed to remain steady throughout the simulation. The performance metrics chosen for the analysis are PDR, throughput, delay, average routing goodput, and MAC/PHY overhead.

Greedy Perimeter Coordinator Routing (GPCR), Zone Routing Protocol (ZRP), VehicleAssisted Data Delivery (VADD), Greedy Perimeter Stateless Routing (GPSR), Inter-Vehicle Geocast (IVG), Geographic-Delay Tolerant Network (Geo-DTN), Contention Based Forwarding (CBF), DV-CAST (Distribution Vehicular broadCAST) are some of the VANET routing protocols for efficient communication. Table 1 presents a comparative study of some routing protocols that can be implemented for the VANET scenario based on the type of protocol, type of communication, routing type, and type of data transmission approach. The GPCR is based on a predetermined path; this protocol implements a restricted greedy forwarding method. Coordinator nodes (nodes on junctions) are preferred over non-coordinator nodes even if the latter is not the geographically closest nodes to the destination. It considers the buffer size of the queue and the distance of nodes from the destination. ZRP offers both proactive and reactive advantages, designed to deal with excess bandwidth of proactive protocol and inefficient reactive protocol flooding. It maintains a topology with a node as the center of each zone. By doing this, routing information is immediately available within each zone. It provides the most efficient routing from the local routing information of the zones for the destinations outside the zone where it uses a route discovery method. VADD provides service using predictable vehicle mobility to implement the concept of carry forward by multihop data delivery. Based on beacon information, the GPSR selects the node closest to the final destination. Depending on the scenario, it selects a node for packet transmission by using greedy forwarding or perimeter forwarding.

Table 1. Classification and Comparison of Routing Protocols.

\begin{tabular}{ccccc}
\hline Protocol Type & Protocol & $\begin{array}{c}\text { Communication } \\
\text { Type }\end{array}$ & Routing Type & $\begin{array}{c}\text { Data } \\
\text { Transmission }\end{array}$ \\
\hline AODV [19] & Topology-based & Uni/Multi-cast & Reactive & Multi-hop \\
DSDV [20] & Topology-based & Uni-cast & Proactive & Multi-hop \\
DSR [21] & Topology-based & Uni-cast & Reactive & Multi-hop \\
OLSR [22] & Topology-based & Uni-cast & Proactive & Multi-hop \\
GPCR [23] & Position-based & Uni-cast & Reactive & Greedy \\
ZRP [24] & Position-based & Uni-cast & Hybrid & Multi-hop \\
VADD [25] & Position-based & Uni-cast & Reactive & Greedy \\
GPSR [26] & Position-based & Uni-cast & Reactive & Multi-hop \\
IVG [27] & Geo-based & Multi-cast & Reactive & Greedy \\
Geo-DTN [28] & Position-based & Uni-cast & Reactive & Multi-hop \\
CBF [29] & Position-based & Multi-cast & Reactive & Multi-hop \\
DV-Cast [30] & Broadcast-based & Broadcast & Proactive & Mredy \\
\hline
\end{tabular}

The IVG geo-cast routing protocol relays safety information, such as an accident, to all nodes that are located on the highway. Timer-based mechanisms allow each node of this protocol to receive packets and rebroadcast them once a timer expires. During periodic broadcasts, the IVG protocol reduces fragmentation between members of multicast groups. Heterogeneous networks like that of VANET can benefit from the DTN routing protocol. DTN protocols utilize the store-carry-forward mechanism for packet forwarding to overcome the challenge of frequent disconnection. The CBF technique is used in Intelligent Transportation Systems (ITS) to disseminate messages for traffic safety applications. This protocol implements a contention mechanism based on the priority of packets. DV-Cast is 
a distributed broadcast routing protocol mostly suited for connected highways; broadcasting happens based on local topological information. The broadcast routing protocols are frequently used in VANET for traffic management, weather monitoring, road conditions, and for advertising and announcements of messages between vehicles.

The protocols like AODV, GPSR, OLSR, DSDV are modified in [31-35] for an efficient adaptive routing protocol implementation of VANET scenario. The optimized routing protocols provide enhanced network performances. In terms of packet delivery ratio, the improved GPSR model [36] performs better than the existing GPSR. A vulnerability attack on the DV-CAST protocol is described, along with safety specifications in secure DVCAST [37]. Authentication, legitimacy, and non-repudiation of safety messages along with vehicle privacy are included in secure DV-CAST, providing less latency than traditional DV-Cast.

\section{Proposed Work}

An efficient routing mechanism in VANETs high mobile environment is necessary for proper data dissemination. Numerous routing protocols have been proposed in the past $[38,39]$ and they are mainly classified as topology-based, position-based, reactivebased, proactive-based, cluster-based, and opportunistic-based. This work compares the performance of OLSR and DSDV proactive protocols and AODV reactive protocol on a realistic VANETs environment. Furthermore, considering the path loss due to obstacles (such as vehicles, buildings, road-curvature, etc.) in the path of signal transmission from one vehicle to another has to be addressed. Loss of data packet depicts network channel characteristics, and propagation models are implemented to estimate the path loss. Thus, path loss and fading models are analysed along with proper routing protocol selection.

The WAVE includes IEEE 802.11p and IEEE 1609.1 4 standards. The IEEE standard implements CSMA/CA with QoS and reliable communication. Thus, as an interfacing protocol for the scenario, like IEEE 802.11p and 1609.x provides efficient short-range communication. A flow diagram of simulation is shown in Figure 2.

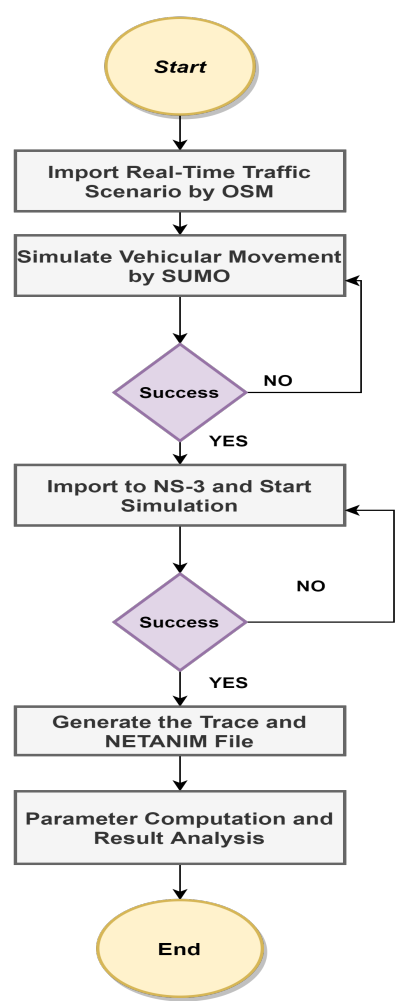

Figure 2. Flow diagram of simulation. 


\subsection{Propagation Models}

The propagation models are essential for determining channel characteristics vital in V2V and V2I communication. The propagation factors affect the signal strength travelling from source to destination in a wireless medium, including path loss and signal fading. For the analysis, this work considers four models: FRIIS (FS) path loss, Two-Ray Ground (TR), Log-Distance path loss (LD), and Nakagami (NK) fading models [40-42]. The pictorial representation of FRIIS and Two-Ray Ground propagation model is shown in Figure 3a and Figure $3 \mathrm{~b}$ respectively.

\subsubsection{FRIIS (Free Space Propagation Model)}

It is one of the earliest propagation models where radio waves move in an omnidirectional pattern from the source used in wireless networks, specifically in MANET. The radio waves move in free space without any obstacles, and the received power is computed upon power transmitted, antenna gain, and sender-receiver distance. This model revolves around a transmission range of the transmitter. If the receiver is present within the transmitter's range, the packet is received; otherwise, it is lost.

$$
P_{r}=\frac{P_{t} G_{t} G_{r} \lambda^{2}}{(4 \pi d)^{2} L}
$$

Here, $P_{t}$ is the transmit power $(\mathrm{W}), P_{r}$ is the reception power $(\mathrm{W}), \lambda$ is the wavelength $(\mathrm{m})$. Transmit and receive gains are $G_{t}$ and $G_{r}$, respectively. The distance between transmitter and receiver is $d$, and system loss is $L$.

As per Equation (1) [43], the received signal strength depends on the strength of the transmitted signal, the receiving and transmitting antenna gain, and the distance between the transmitter and the receiver.

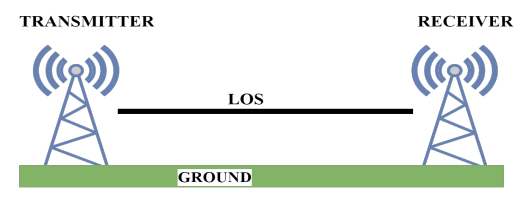

(a)

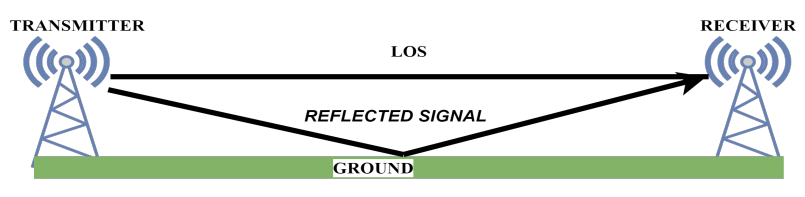

(b)

Figure 3. (a) FRIIS and (b) Two-Ray Ground propagation models [44].

\subsubsection{Two-Ray Ground Propagation Model}

This model is one of the most common models used in VANETs for multipath radio propagation and is also an ideal model for many wireless applications. As stated in [42], this model has a more practical implication than FRIIS as it considers the direct path and the ground reflected direction. It predicts the path loss between the transmitter and receiver antenna within the Line-of-Sight (LOS) with nodes present in a planar structure.

$$
P_{r}=\frac{P_{t} G_{t} G_{r}\left(H_{t}^{2} * H_{r}^{2}\right)}{d^{4} * L}
$$

The reception power, the transmitter and receiver antenna's height, and the distance through which the antenna's receive and transmit are signified by $P_{r}, H_{t}$ and $H_{r}$, and $d$, respectively. $G_{t}$ and $G_{r}$ denotes the antenna's gain of receiving and the transmitting, respectively, with $L$ as system Loss. An oscillation parameter is induced in this model by the ray's constructive and destructive combination, making it unsuitable for shorter distances. 


\subsubsection{Log-Distance Propagation Model}

This model is suitable for propagation in dense areas. The model estimates the path loss for a more considerable distance and communication range. This model computes an exponential path loss incurred from a distance between a transmitter and a receiver [42]. The model has assumed an exponential path loss over the transmitter's and receiver's distance.

$$
L=L_{0}+10 n \log \left(\frac{d}{d_{0}}\right)
$$

Here, $L$ denotes the path loss, $d$ the distance, the reference distance is denoted by $d_{0}$, with path loss for reference distance denoted by $L_{0}$ and $n$ states the path loss exponent.

\subsubsection{Nakagami Fading Model}

It is primarily a fading model that accounts for signal strength variation caused by multi-path fading. As the model is not very suitable as a path loss model for simulations, it can be used with other loss models for a better performance result. It has continuous probability distribution for the varied environments, including gamma distribution [45].

$$
p(x ; m, \omega)=\frac{2 m^{m}}{\Gamma(m) \omega^{m}} x^{2 m-1} e^{-\frac{m}{\omega} x^{2}}
$$

Here, $m$ parameter denotes the fading depth and $\omega$-the average power received. The probability density function (pdf) at a given distance is computed by modifying gamma distributions. The signal follows gamma distribution as a stochastic model with average power fading and severity with amplitude $x>=0$. For $m=1$, the Nakagami becomes the Rayleigh model.

\subsection{Routing Protocols}

Proper routing is a challenge due to highly dynamic topology, varied network characteristics, and different types of communication (V2V, V2I). The continuous vehicular movement demands a suitable communication link. The primary requirement of an efficient routing protocol is to provide QoS, better traffic and network management, handle mobility and provide delay-tolerant message delivery. The three protocols considered are topology-based, where routing information is mainly stored in the routing table. An efficient routing mechanism is necessary for appropriate route selection from sender to receiver via a proper communication link.

\subsubsection{Ad hoc On-Demand Distance Vector (AODV)}

The name depicts it as an on-demand routing protocol with a conventional routing table, with a timer set for each node. If routing is not performed within a specific time frame, the node is removed from the table. The source, as well as intermediate nodes, carries all neighbours' information and messages. A hello message is sent for communication, and a route is discovered by sending Route Request Message (RREQ).

\subsubsection{Optimized Link State Routing (OLSR)}

The protocol is a proactive type depending on routing table for data access; it allows data dissemination by connecting nodes with a hello message and link-state information. The next-hop is computed based on the shortest forwarding path. Then each node rebroadcasts link-state information and also tracks information from other nodes. 


\subsubsection{Destination-Sequenced Distance Vector (DSDV)}

The protocol uses hop-by-hop routing and broadcasts updates periodically. It is based on Bellman-Ford routing mechanism; each node consists of a routing table listing the destination nodes, hops to reach each node, and sequence numbers of the information received. The mechanism avoids routing loops' formation as the node's location and its transmission information are broadcast to all nodes. When a node transmits, it updates its neighbours, allowing them to recognize that they are one hop from the source and update their distance vector information. In this manner, every node stores the information of the next routing hop in the routing table.

\subsection{Interfacing Protocols}

This analysis incorporates the IEEE 802.11p PHY/MAC protocol with multichannel extensions based on IEEE 1609 WAVE standard.

\subsubsection{IEEE $802.11 \mathrm{p}$}

For vehicular communication, IEEE $802.11 \mathrm{p}$ and $802.11 \mathrm{~b}$ are widely used for wireless connectivity. IEEE 802.11p is considered as the cross-layer standard of PHY/MAC, incorporating OFDMA and frequency band specified for vehicular communication of $5.9 \mathrm{GHz}$. The IEEE 802.11p MAC is designed based on CSMA/CA, i.e., Carrier Sense Multiple Access with Collision Avoidance. A node initially senses the channel before transmission, and during the transmission process, the node and channel become inactive for a Short Inter-frame Space (SIFS). The transmission resumes only when the channel is idle. In the case of a busy channel, an arbitrary back-off mechanism is implemented with Contention Window $(\mathrm{CW})$ of $[0, \max (\mathrm{CW})]$ and starts to transmit only at the lapse of the back-off timer. As part of the multi-channel access mechanism, the Control Channel (CCH) is dedicated to safety messages, and the Service Channel $(\mathrm{SCH})$ is for non-safety communications. Control packets broadcast throughout the $\mathrm{CCH}$ with no acknowledgment required from the receiver. However, in $\mathrm{SCH}$, the packets are retransmitted if no acknowledgment is received within the time frame.

\subsubsection{IEEE 1609 WAVE}

This protocol includes network and resource management supporting multi-channel operation with network security and administration. IEEE 1609 comprises four standards: IEEE 1609.1 for application management, IEEE 1609.2 for safety and security mechanisms, IEEE 1609.3 WAVE management and networking services, and IEEE 1609.4 for multichannel operations with logical connections and controls of each layer.

\section{Performance Metric and Simulation Setup}

This section presents the chosen performance metric for evaluation and simulation setup. Results are presented and discussed in detail.

\subsection{Performance Evaluation Metrics}

The performance metrics taken for comparative analysis are discussed below. The summary of the used notations is shown in Table 2. 
Table 2. Summary of notations.

\begin{tabular}{|c|c|}
\hline Notation & Description \\
\hline$T h_{a v g}$ & Average Throughput \\
\hline$P_{r}$ & Packet Received \\
\hline$P_{S}$ & Packet Sent \\
\hline$T_{l}$ & Time of last received packet \\
\hline$T_{f}$ & Time of first transmit packet \\
\hline EED & End-to-End Delay \\
\hline$T_{\text {delay }}$ & Transmission Delay \\
\hline$P_{\text {delay }}$ & Propagation Delay \\
\hline Proc delay $_{1}$ & Processing Delay \\
\hline$Q_{\text {delay }}$ & Queuing Delay \\
\hline PDR & Packet Delivery Ratio \\
\hline Goodput $_{A v g}$ & Average Goodput \\
\hline$T_{\text {Sim }}$ & Total Simulation Time \\
\hline$M A C-P H Y_{o h}$ & MAC-PHYsical Overhead \\
\hline$P H Y_{S}$ & PHYsical layer packets send in bytes \\
\hline$A P P_{S}$ & Application layer packets send in bytes \\
\hline$P H Y_{d}$ & PHYsical layer packets delivered in bytes \\
\hline
\end{tabular}

\subsubsection{Average Throughput}

Throughput is the rate of successful packet delivery over a communication channel. The computation is done by dividing the total bits transmitted over a link per given unit of time or the number of received packets by the last received packet minus the first transmitted packet. In Equation (5), the number 8 indicates the packets received converted to bits. It is usually measured in bps, Kbps, or Mbps. High throughput states better network performance.

$$
T h_{\text {avg }}=\left(P_{r} * 8\right) /\left(T_{l}\right)-\left(T_{f}\right)
$$

\subsubsection{End-to-End Delay}

End-to-end delay refers to the sum of all delays in the link caused by intermediate nodes-the total time taken by a packet from sender to receiver vehicle. It is computed as the sum of all communication delays. It is measured in sec, ms, or ns. A less delay infers better network performance.

$$
E E D=\left(T_{\text {delay }}+P_{\text {delay }}+Q_{\text {delay }}+\text { Proc delay }_{\text {d }}\right) / \text { total }_{r}
$$

\subsubsection{Packet Delivery Ratio}

The ratio of the packets received by other vehicles to those sent out by the transmitting vehicle. Higher PDR values indicate improved network performance.

$$
P D R=\text { Total }_{r} / \text { TotalPs }
$$

\subsubsection{Average Routing Goodput}

Goodput is the value of throughput computed in the application layer. It considers only the Basic Safety Message (BSM) or the useful data for computation rather than all data as in the case of throughput. In VANETs, BSM are considered the most useful information. 
They are measured in bps, Kbps, or Mbps. The more the Goodput, the better is the performance of the network.

$$
\text { Goodput }_{\text {Avg }}=\text { TotalP }_{r} * 8 / \text { TotalT }_{\text {Sim }} / 1000
$$

\subsubsection{MAC/PHY Overhead}

More packets can be sent and received with a more extensive network, causing overhead in packet routing. It is also termed as the saturation degree of a network. It measures the scalability of the network, i.e., if the overhead does not increase with the increase of vehicles number, then network performance is good. It depends on the total WAVE BSM sent. The bandwidth of the network is shared for routing as well as application packets. Less overhead infers better network performance.

$$
M A C-P H Y_{o h}=\text { totalPHY }- \text { total } A P P_{s} /\left(\text { total } P H Y_{d}\right) .
$$

\subsection{Simulation Parameters Settings}

A proper selection of routing mechanisms with propagation models can enhance the performance in V2I and V2V communication. Based on varying numbers of nodes and simulation times, routing protocols and propagation models from the perspective of throughput, end-to-end delay, packet delivery ratio, average routing throughput, and MAC/PHY overhead is examined. The parameter settings for the scenario taken for simulation are depicted in Table 3.

Table 3. Simulation parameters setting.

\begin{tabular}{cc}
\hline Simulator & NS-3.29, SUMO \\
\hline Radio Range & DSRC \\
MAC Protocol & $802.11 \mathrm{p}$ \\
Routing Protocols & AODV, DSDV, OLSR \\
Propagation Model & FRIIS, Two Ray Ground, Log-Distance, Nakagami \\
Channel Type & Wireless \\
Vehicle Speed & Random \\
Transmission range & $145 \mathrm{~m}$ \\
Transmitting Power & $20 \mathrm{dBm}$ \\
Data Rate & $2048 \mathrm{bps}$ \\
Packet Size & $200 \mathrm{Bytes}$ \\
Simulation time & $300 \mathrm{~s}$ in SUMO \\
Simulation time & $20,40,60,100,120(\mathrm{~s})$ in NS3 \\
Number of Nodes & $20,30,40,50,60,70$ \\
Frequency & $5.9 \mathrm{GHz}$ \\
\hline
\end{tabular}

\subsection{Generation of Simulation Environment}

The target location is downloaded from the Open Street Map (OSM), which refers to the realistic scenario of Rourkela. The .osm file generated is interfaced to SUMO to get the mobility.tcl with details of each node (vehicles), including the number of vehicles, position, speed, and direction.

The simulation is conducted in NS-3 with the mobility.tcl file. To evaluate the environment, the $802.11 \mathrm{p} \mathrm{MAC/PHY,} \mathrm{frequency} \mathrm{of} 5.9 \mathrm{GHz}$, is kept constant for sending Basic Safety Messages (BSM). The network performance evaluation is done by varying propagation models, routing protocols based on simulation time, and the number of nodes (vehicles). A snapshot of the realistic scenario with the simulation SUMO scenario and NetAnim generated is depicted in Figure 4. 


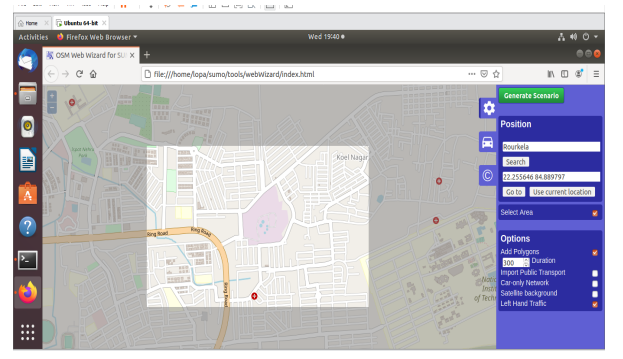

(a)

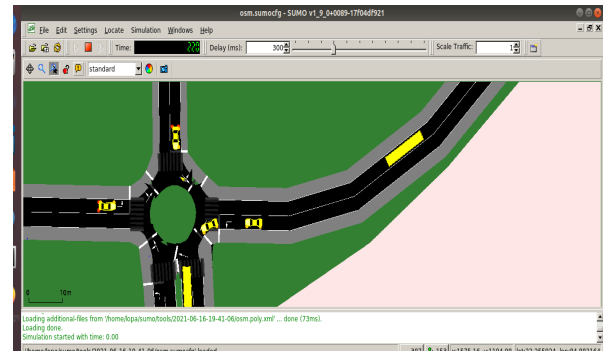

(b)

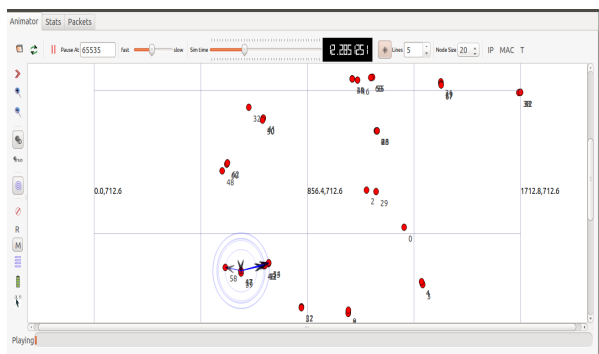

(c)

Figure 4. VANETs Traffic Scenario. (a) Map of Rourkela by OSM; (b) Simulation in SUMO; (c) NetAnim Representation.

\subsection{Results}

The simulation is conducted for propagation models and routing protocols over IEEE 802.11p stack with varying simulation time and number of nodes(vehicles). Based on the varying number of nodes and simulation time, the specified routing protocols and propagation models are examined and the performance metrics such as packet delivery ratio, throughput, delay, goodput, and overhead are discussed. Various scenarios taken for the analysis of network performance are given below.

\subsubsection{Comparative Analysis 1}

This case considers a static scenario with propagation model (TK) and simulation time 20 s, node speed 20 m/s, mobility model Random-Way Point as constant factors. The transmit power and transmission range of each node are also fixed. The routing protocols are compared based on the performance metrics varying the number of nodes. Figure 5a depicts OLSR outperforms AODV and DSDV in terms of end-to-end delay. For packet delivery ratio and throughput as shown in Figure 5b,c OLSR shows better performance with the increase in the number of nodes. Thus, overall, the performance of OLSR is better than AODV and DSDV in terms of throughput, end-to-end delay, and packet delivery ratio.

\subsubsection{Comparative Analysis 2}

This case is based on the comparison of the propagation models with constant routing protocol, interfacing protocol, and varying simulation time. The realistic scenario is taken with 71 vehicles communicating by sending BSM. Figure 6 shows that the Nakagami fading model has the worst performance of all. FRIIS and the Two-Ray Ground model show similar performance. The Log-Distance model shows minimum delay and maximum packet delivery ratio depicted in Figure 6a,b. MAC/PHY overhead is highest in the case of the Nakagami model and lowest for FRIIS and Two-Ray Ground, as shown in Figure 6e. FRIIS and Two-Ray Ground show high throughput, which drastically increases after a specific time and shows better average routing goodput compared to the other two models as shown in Figure $6 c, d$, respectively. Finally, to sum up, the Log-Distance outperforms other models in terms of end-to-end delay and packet delivery ratio. Still, FRIIS and Two-Ray perform better for throughput and average routing goodput as simulation time increases. 


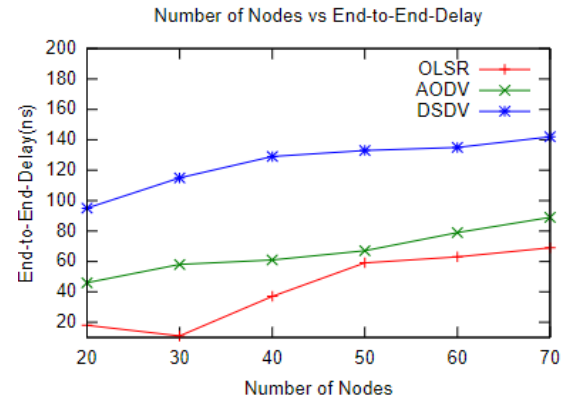

(a)

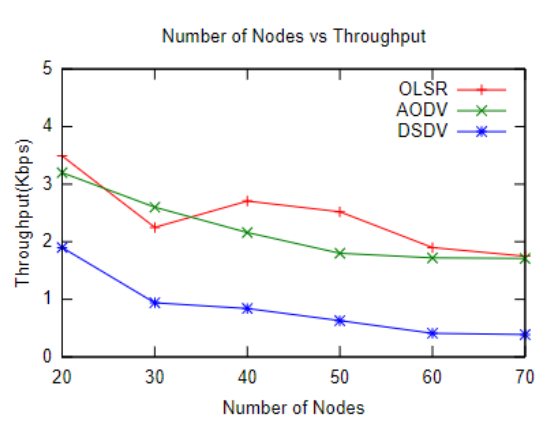

(c)

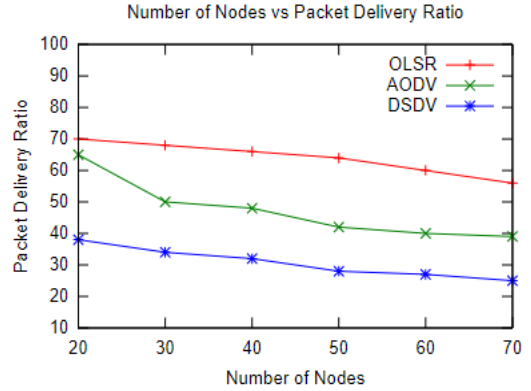

(b)

Figure 5. Performance Analysis of Routing Protocols varying Node Numbers for static scenario. (a) Number of Nodes vs. End-to-End Delay; (b) Number of Nodes vs. Packet Delivery Ratio; (c) Number of Nodes vs. Throughput.

\subsubsection{Comparative Analysis 3}

This scenario is based on a comparison of propagation models with constant routing protocol and interfacing protocol with varying numbers of nodes for a fixed time of $60 \mathrm{~s}$. The end-to-end delay is minimum for the Log-Distance model compared to other models as per Figure 7a. However, Two-Ray Ground and FRIIS outperform other models in terms of packet delivery ratio, throughput, average routing goodput, with the smallest value of MAC/PHY overhead depicted in Figure 7b-e, respectively.

\subsubsection{Comparative Analysis 4}

This analysis is performed taking propagation model (TK) with the constant routing protocol. The simulation is run for $60 \mathrm{~s}$ with varying numbers of nodes. Based on the performance metrics specified, Figure 8 shows a graphical comparison of the protocols. From the graphs, the result infer that OLSR performs high on end-to-end delay, throughput, packet delivery ratio, and average routing goodput with very little MAC/PHY overhead compared to other protocols. 


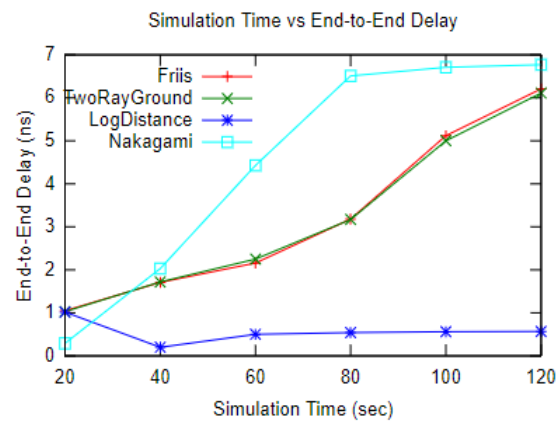

(a)

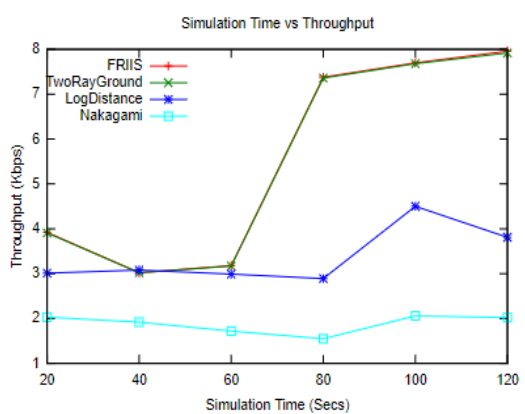

(c)

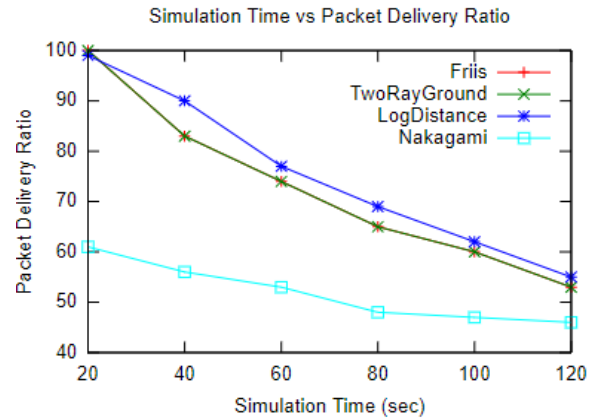

(b)

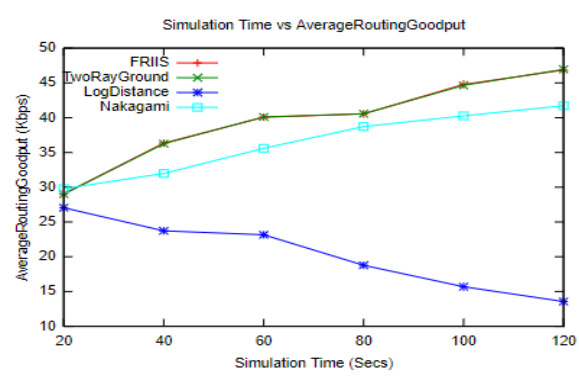

(d)

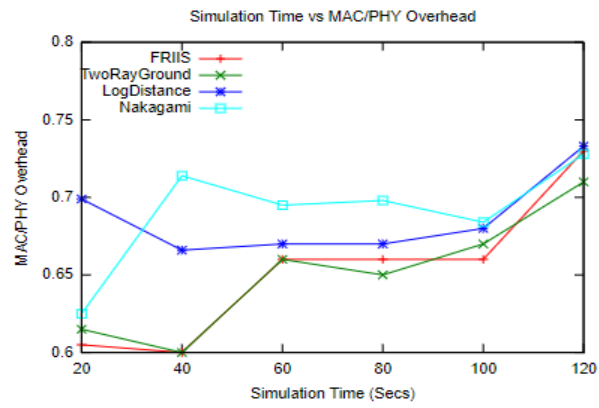

(e)

Figure 6. Performance Analysis of Propagation Model varying Simulation Time. (a) Simulation Time vs. End-to-End Delay; (b) Simulation Time vs. Packet Delivery Ratio; (c) Simulation Time vs. Throughput; (d) Simulation Time vs. AverageRoutingGoodput; (e) Simulation Time vs. MAC/PHY Overhead. 


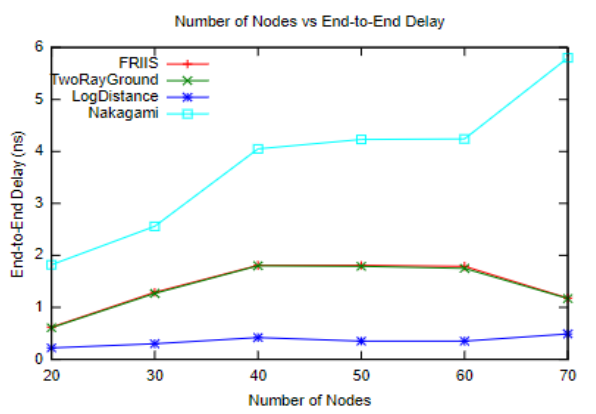

(a)

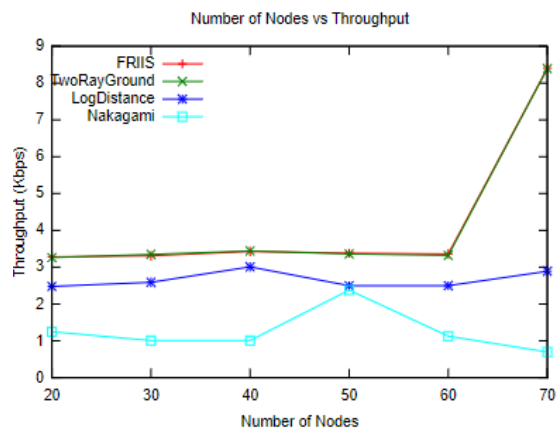

(c)

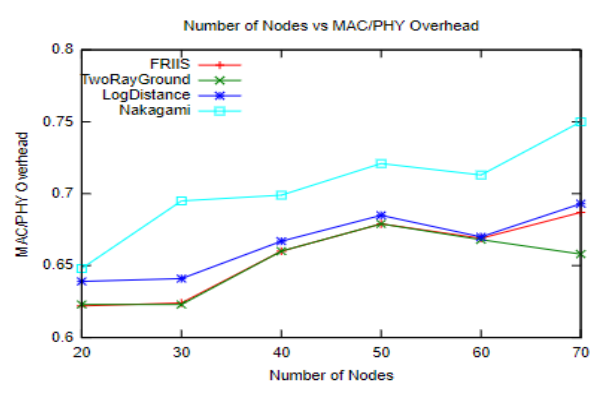

(e)

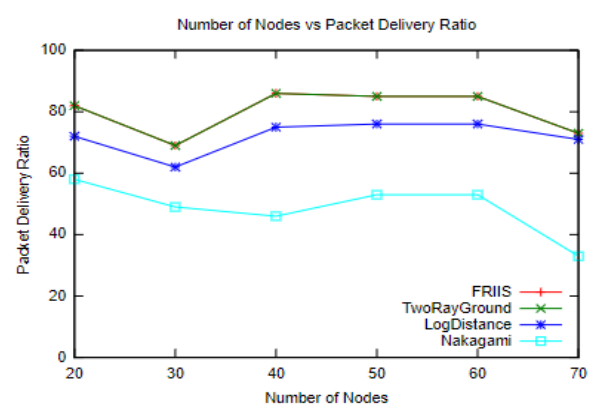

(b)

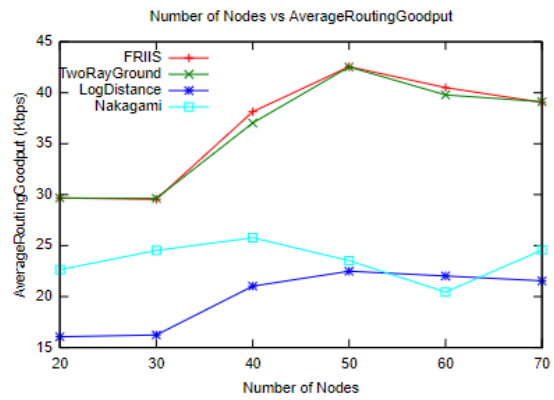

(d)

Figure 7. Performance Analysis of Propagation Model varying Number of Nodes. (a) Number of Nodes vs. End-to-End Delay; (b) Number of Nodes vs. Packet Delivery Ratio; (c) Number of Nodes vs. Throughput; (d) Number of Nodes vs. AverageRoutingGoodput; (e) Number of Nodes vs. MAC/PHY Overhead. 


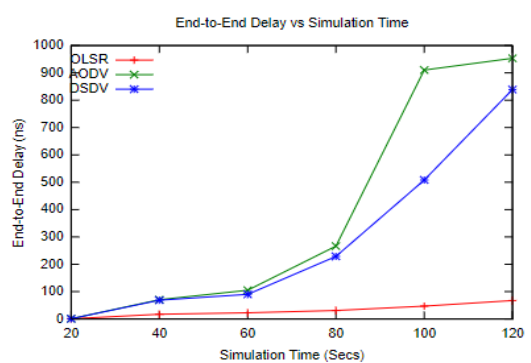

(a)

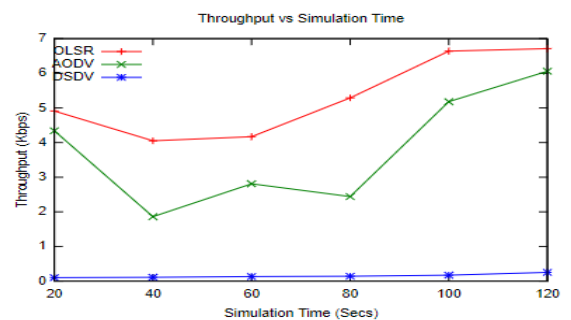

(c)

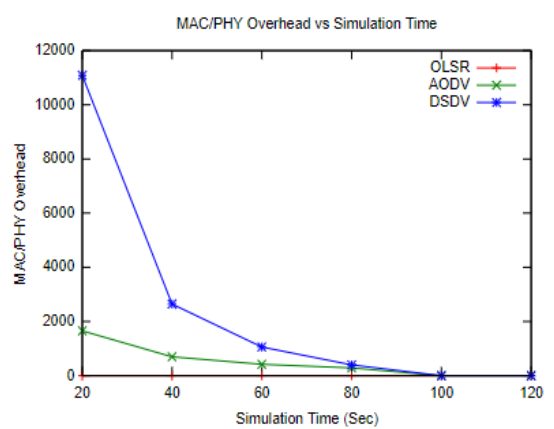

(e)

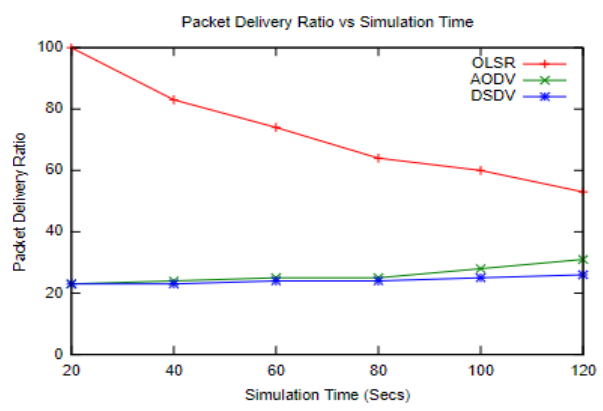

(b)

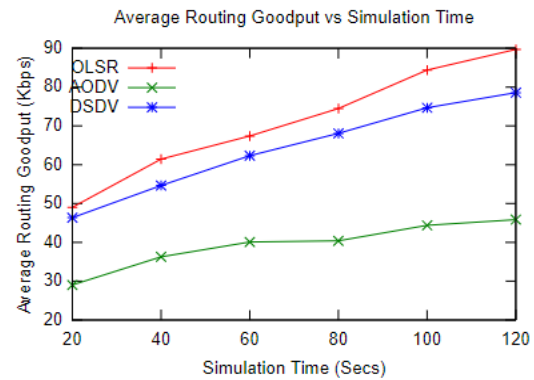

(d)

Figure 8. Performance Analysis of Routing Protocols varying Simulation Time. (a) Simulation Time vs. End-to-End Delay; (b) Simulation Time vs. Packet Delivery Ratio; (c) Simulation Time vs. Throughput; (d) Simulation Time vs. AverageRoutingGoodput; (e) Simulation Time vs. MAC/PHY Overhead.

\subsubsection{Comparative Analysis 5}

The propagation model (TK) being constant, routing protocols are compared in this case. The simulation is run for varying simulation times for realistic scenario with 71 vehicles communicating for BSM transmission. The comparative analysis graph depicted in Figure 9 shows that OLSR outperforms AODV and DSDV in terms of throughput, endto-end delay, packet delivery, average routing goodput, MAC/PHY overhead despite an increase in simulation time. 


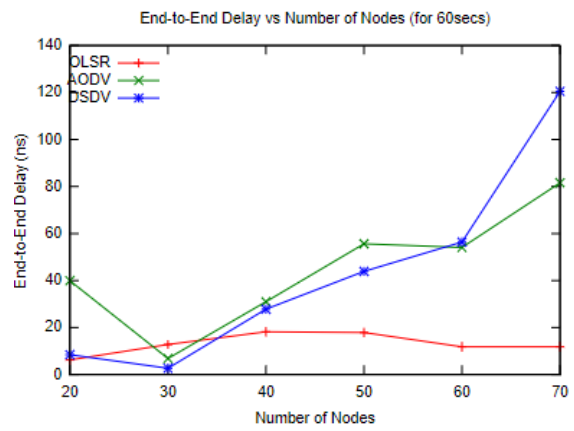

(a)

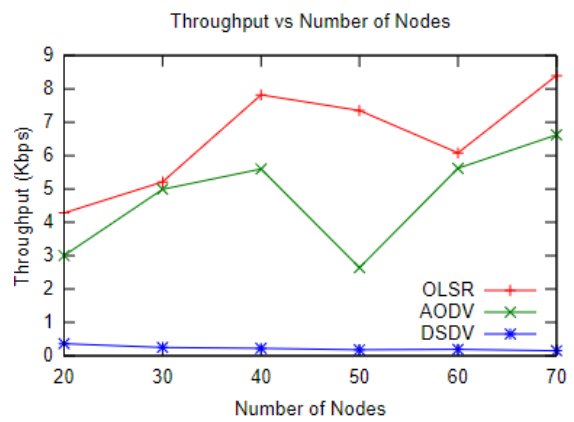

(c)

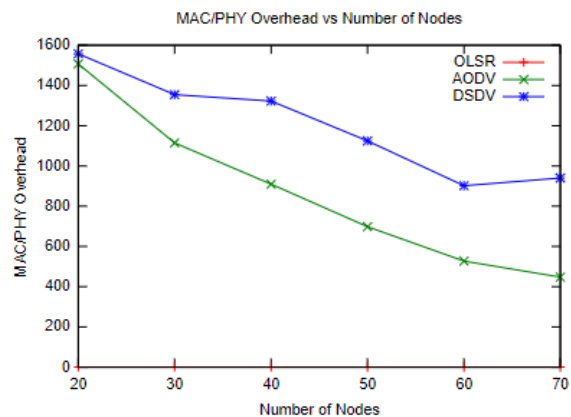

(e)

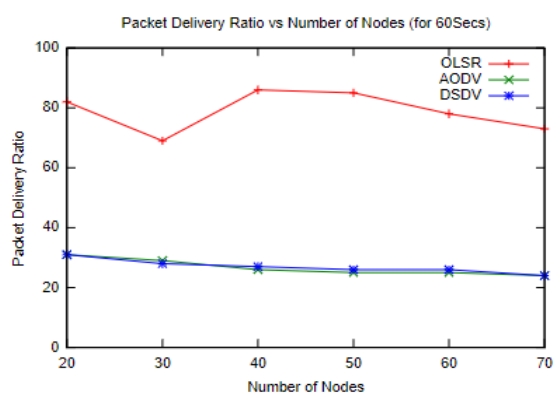

(b)

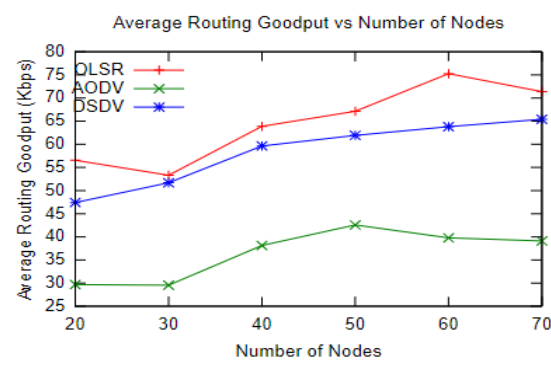

(d)

Figure 9. Performance Analysis of Routing Protocols varying Number of Nodes for Realistic Scenario simulated for 60 s. (a) Number of Nodes vs. End-to-End Delay; (b) Number of Nodes vs. Packet Delivery Ratio; (c) Number of Nodes vs. Throughput; (d) Number of Nodes vs. AverageRoutingGoodput; (e) Number of Nodes vs. MAC/PHY Overhead.

\subsection{Summary of Comparative Study}

To recapitulate the comparative analysis, in scenario 1 with the Two-Ray propagation model and routing protocols states OLSR has better performance than AODV and DSDV. As per scenario 2, the Log-Distance model outperforms other propagation models in terms of delay and packet delivery, whereas FRIIS and Two-Ray give better performance in terms of throughput, average routing goodput, and MAC/PHY overhead. In scenario 3, Log-Distance has less delay, however, Two-Ray and FRIIS outperform in terms of packet delivery, throughput, average goodput, and overhead. It is to be noted that although Nakagami is not the best propagation model, it gives the most stable performance. Further, FRISS and Two-Ray are deterministic propagation models, whereas Nakagami and LogDistance are probabilistic models. Thus, using models like FRISS and Two-Ray may not be feasible for varying scenarios as the results are misleading. For real-time scenarios, models like Nakagami and Log-Distance can provide an accurate result. A more appropriate model can be a combination of Nakagami (fading model) and Log-Distance (path-loss propagation 
model). As per analysis 4, OLSR performs better in terms of delay, throughput, packet delivery, goodput, and overhead compared to AODV and DSDV. The comparative analysis 5, for scenario 5, depicts OLSR to perform better than the other compared protocols in terms of all performance metrics. To sum up, OLSR is the best routing protocol than the compared ones for a VANET scenario due to its multi-point relay mechanism for a more efficient, organized, reliable, and scalable routing. Whereas, the preferred propagation model is Log-Distance and Nakagami rather than FRIIS or Two-Ray, as it shows the most accurate representation and better performance.

\section{Conclusions and Future Work}

This paper has presented the performance analysis of VANETs propagation models and routing protocols. In this work, SUMO and NS-3.29 are used for simulation. The results are presented and discussed in detail. Results show that the OLSR has a large throughput, end-to-end delay, packet delivery ratio, and MAC/PHY overhead over AODV and DSDV, as analysed using a Two-Ray Ground Propagation model. Whereas, taking OLSR with a different propagation model proved that the performance of FRIIS and Two-Ray Ground are nearly the same, and the Log-Distance propagation model outperforms the others with varying simulation time. With the varying number of nodes (vehicles), the packet delivery ratio and throughput of FRIIS and Two-Ray Ground proved to be better than Log-Distance but the most stable model is Nakagami. Therefore, the most appropriate model for a real-time VANET scenario is Log-Distance and Nakagami rather than FRISS and Two-Ray. Analysis and results may provide guidelines for future implementation and design of traffic control mechanisms for safety applications and faster data dissemination. The analysis presented proved that the propagation model is an essential factor for the performance of a network.

Future work focuses on testing other mobility models like Random-Way Point, Manhattan, Gaussian, with some contemporary routing protocols. Furthermore, a model enhances the MAC layer 802.11p for delay-tolerant delivery of safety messages and control congestion schemes.

Author Contributions: Conceptualization, L.H. and A.K.; methodology, L.H. and A.K.; software, L.H., A.K. and B.P.N.; validation, L.H., A.K. and B.P.N.; formal analysis, L.H., A.K., B.P.N., G.G.M.N.A. and B.S.; investigation, L.H., A.K., B.P.N. and B.S.; writing-original draft preparation, L.H. and A.K.; writing-review and editing, L.H., A.K., B.P.N., G.G.M.N.A. and B.S. All authors have read and agreed to the published version of the manuscript.

Funding: This research received no external funding.

Institutional Review Board Statement: Not applicable.

Informed Consent Statement: Not applicable.

Data Availability Statement: All data generated and analyzed during this study are included in this published article.

Conflicts of Interest: The authors declare no conflict of interest.

$\begin{array}{ll}\text { List of Acronyms } \\ \text { Acronym } & \text { Description } \\ \text { AODV } & \text { Ad-hoc On-demand Distance Vector } \\ \text { AOMDV } & \text { Ad-hoc On-demand Multipath Distance Vector Routing protocol } \\ \text { BSM } & \text { Basic Safety Message } \\ \text { CSMA/CA } & \text { Carrier-Sense Multiple Access with Collision Avoidance } \\ \text { CCH } & \text { Control Channel } \\ \text { DSDV } & \text { Destination Sequenced Distance Vector } \\ \text { DSR } & \text { Dynamic source routing protocol } \\ \text { DSRC } & \text { Dedicated Short-Range Communication }\end{array}$




$\begin{array}{ll}\text { DYMO } & \text { Dynamic Manet on demand } \\ \text { FCC } & \text { Federal Communication Commission } \\ \text { FRIIS } & \text { Free Space Propagation Model } \\ \text { GPCR } & \text { Greedy Perimeter Coordinator Routing } \\ \text { GPSR } & \text { Greedy Perimeter Stateless Routing } \\ \text { GTS } & \text { Global Transportation System } \\ \text { ITS } & \text { Intelligent Transportation System } \\ \text { LTE } & \text { Long-Term Evolution } \\ \text { MMPR-OLSR } & \text { Minimum Multi-Point Relay Optimal Link State Routing } \\ \text { MAC/PHY } & \text { Media Access Control/PHYsical } \\ \text { OFDMA } & \text { Orthogonal Frequency Division Multiple Access } \\ \text { QoS } & \text { Quality of Service } \\ \text { PDR } & \text { Packet Delivery Ratio } \\ \text { SCH } & \text { Service CHannel } \\ \text { SIFS } & \text { Short Inter-frame Space } \\ \text { SUMO } & \text { Simulation of Urban MObility } \\ \text { WLAN } & \text { Wireless Local Area Network } \\ \text { WAVE } & \text { Wireless Access for Vehicular Environment }\end{array}$

\section{References}

1. Sultan, A.S.; Al-Doori, M.M.; Al-Bayatti, H.A.; Zedan, H. A Comprehensive Survey on Vehicular Ad hoc Network. J. Netw. Comput. Appl. 2014, 37, 380-392. [CrossRef]

2. Hadded, M.; Muhlethaler, P.; Laouiti, A.; Zagrouba, R.; Saidane, A.L. TDMA-based MAC Protocols for Vehicular Ad hoc Networks: A Survey, Qualitative Analysis, and Open Research Issues. IEEE Commun. Surv. Tutor. 2014, 17, 2461-2492. [CrossRef]

3. Moharrum, A.M.; Al-Daraiseh, A.A. Toward Secure Vehicular Ad-hoc Networks: A Survey. IETE Tech. Rev. 2012, 29, 80-89. [CrossRef]

4. Usha, M.; Ramakrishnan, B. A Robust Architecture of the OLSR Protocol for Channel Utilization and Optimized Transmission using Minimal Multi Point Relay Selection in VANET. Wirel. Pers. Commun. 2019, 109, 1-25. [CrossRef]

5. Lopez, A.P.; Behrisch, M.; Bieker-Walz, L.; Erdmann, J.; Pang, Y. Microscopic Traffic Simulation using SUMO. In Proceedings of the IEEE Intelligent Transportation Systems Conference (ITSC), Maui, HI, USA, 4-7 November 2018.

6. Priyambodo, K.T.; Wijayanto, D.; Gitakarma, S.M. Performance Optimization of MANET Networks through Routing Protocol Analysis. Computers 2020, 10, 2. [CrossRef]

7. Guillen-Perez, A.; Montoya, M.A.; Sanchez-Aarnoutse, C.J.; Cano, D.M. A Comparative Performance Evaluation of Routing Protocols for Flying Ad-Hoc Networks in Real Conditions. Appl. Sci. 2021, 11, 4363. [CrossRef]

8. Shuhaimi, I.N.; Ashmadi, L.N.; Abdullah, E.; Mohamad, R.; Mohamad, Y.S. Performance Analysis of Radio Propagation Models in VANET Application. In Proceedings of the 11th IEEE Symposium on Computer Applications and Industrial Electronics (ISCAIE), Penang, Malaysia, 3-4 April 2021; pp. 372-377.

9. Yogarayan, S.; Razak, A.F.S.; Abdullah, A.F.M.; Ibrahim, Z.S.; Raman, J.K. A Review of Routing Protocols for Vehicular Ad-Hoc Networks (VANETs). In Proceedings of the 8th International Conference on Information and Communication Technology (ICoICT), Yogyakarta, Indonesia, 24-26 June 2020; pp. 1-7.

10. Freitas, P.E.; Heimfarth, T.; Wagner, R.F.; Pereira, E.C.; Larsson, T. Exploring geographic context awareness for data dissemination on mobile ad hoc networks. Ad Hoc Netw. 2013, 11, 1746-1764. [CrossRef]

11. Narayan, S.A.; Reddy, R.R.; Josephin, J.F. Secured Congestion Control in VANET Using Greedy Perimeter Stateless Routing (GPSR). In Artificial Intelligence and Evolutionary Computations in Engineering Systems; Springer: Berlin/Heidelberg, Germany, 2020; pp. 683-700.

12. Wu, L.; Wang, X. Performance Analysis of CBRP, AODV and DSR Routing Protocols in VANETs Based on IDM-IM. In International Conference on Communicatins and Networking in China; Springer: Berlin/Heidelberg, Germany, 2017; pp. 33-40.

13. Benmir, A.; Korichi, A.; Bourouis, A.; Alreshoodi, M.; Al-Jobouri, L. An Enhanced GPSR Protocol for Vehicular Ad hoc Networks. In Proceedings of the 11th Computer Science and Electronic Engineering (CEEC), Colchester, UK, 18-20 September 2019; pp. 85-89.

14. Alamsyah; Setijadi, E.; Purnama, E.K.I.; Pumomo, H.M. Performance Comparative of AODV, AOMDV and DSDV Routing Protocols in MANET Using NS2. In Proceedings of the International Seminar on Application for Technology of Information and Communication, Semarang, Indonesia, 21-22 September 2018; pp. 286-289.

15. Sallum, A.E.E.; Santos, D.G.; Alves, M.; Santos, M.M. Performance Analysis and Comparison of the DSDV, AODV and OLSR Routing Protocols under VANETs. In Proceedings of the 16th International Conference on Intelligent Transportation Systems Telecommunications (ITST), Lisboa, Portugal, 15-17 October 2018; pp. 1-7.

16. Amina, B.; Mohamed, E. Performance Evaluation of VANETs Routing Protocols using SUMO and NS3. In Proceedings of the IEEE 5th International Congress on Information Science and Technology (CiSt), Marrakech, Morocco, 21-27 October 2018; pp. 525-530. 
17. Angeles, W.; Borin, P.V.; Munaretto, A.; Fonseca, M. The Impact of Propagation Models in the Performance of Ad Hoc Routing Protocols for Urban VANET. In Proceedings of the IEEE 84th Vehicular Technology Conference (VTC-Fall), Montreal, QC, Canada, 18-21 September 2016; pp. 1-5.

18. Tang, F.; Bomin, M.; Nei, K.; Guan, G. Comprehensive Survey on Machine Learning in Vehicular Network: Technology, Applications and Challenges. IEEE Commun. Surv. Tutorials. 2021, 23, 2027-2057. [CrossRef]

19. Venkateswarlu, K.; Murali, G. Performance Evaluation of Dsdv, Aodv Routing Protocols In Vanet. Int. J. Res. Eng. Technol. 2015, 4, 33-37.

20. He, G. Destination-Sequenced Distance Vector Protocol. In Networking Laboratory; Helsinki University of Technology: Espoo, Finland, 2002; pp. 1-9.

21. Pereira, E.E.A.; Leonardo, E.J. Performance of DSR Algorithm in VANETs. In Proceedings of the IEEE 10th Latin-American Conference on Communications (LATINCOM), Guadalajara, Mexico, 14-16 November 2018; pp. 1-6. [CrossRef]

22. Toutouh, J.; José, N.G.; Enrique, A. Intelligent OLSR routing protocol optimization for VANETs. IEEE Trans. Veh. Technol. 2012, 61, 1884-1894. [CrossRef]

23. Li, M.; Gu, Z.; Long, Y.; Shu, X.; Rong, Q.; Ma, Z.; Shao, X. W-GPCR Routing Method for Vehicular Ad Hoc Networks. Sensors 2020, 20, 3406. [CrossRef] [PubMed]

24. Chen, S.Y.; Lin, W.Y. A mobicast routing protocol with carry and forward in vehicular adhoc networks. Int. J. Commun. Syst. 2014, 27, 14161440. [CrossRef]

25. Zhao, J.; Cao, G. VADD: Vehicle-assisted data delivery in vehicular ad hoc networks. IEEE Trans. Veh. Technol. 2008, 57, 1910-1922. [CrossRef]

26. Yang, X.; Li, M.; Qian, Z.; Di, T. Improvement of GPSR Protocol in Vehicular Ad Hoc Network. IEEE Access 2018, 6, 39515-39524. [CrossRef]

27. Hadi, A.; Nasser, T.; Amin, R.; Masoud, A.; Maghsoud, A. Usefulness of multicast routing protocols for vehicular Ad-hoc networks. In Proceedings of the 6th International Symposium on Telecommunications, Tehran, Iran, 6-8 November 2012. [CrossRef]

28. Cheng, P.C.; Lee, K.C.; Gerla, M.; Harri, J. GeoDTN+Nav: Geographic DTN Routing with Navigator Prediction for Urban Vehicular Environments. Mob. Netw. Appl. 2010, 15, 61-82. [CrossRef]

29. Chavan, E.; Roopa, M. Automatic crash guard for motorcycles. Int. J. Electric. Eng. Technol. (IJEET) 2020, 11, 17-26.

30. Tonguz, O.K.; Wisitpongphan, N.; Bai, F. DV-CAST: A distributed vehicular broadcast protocol for vehicular ad hoc networks. Wirel. Commun. 2010, 17, 47-57. [CrossRef]

31. Manel, K.; Lamia, C. SODV speed based ad hoc on demand vector link routing protocol: A routing protocol for VANET networks. In Proceedings of the 2017 Sixth International Conference on Communications and Networking (ComNet), Xi'an, China, 17-19 August 2017; pp. 1-8.

32. Shen, X.; Wu, Y.; Xu, Z.; Lind, X. AODV-PNT: An improved version of AODV routing protocol with predicting node trend in VANET. In Proceedings of the 7th IEEE/International Conference on Advanced Infocomm Technology, Fuzhou, China, 14-16 November 2014; pp. 91-97.

33. Smiri, S.; Ben Abbou, A.; Boushaba, A.; Zahi, A.; Ben Abbou, R. WA-GPSR: Weight-Aware GPSR-Based Routing Protocol for VANET. Int. J. Interact. Mob. Technol. 2021, 15, 69-83. [CrossRef]

34. Kadadha, M.; Otrok, H.; Barada, H.; Al-Qutayri, M.; Al-Hammadi, Y. A Stackelberg Game for Street-Centric QoS-OLSR Protocol in Urban Vehicular Ad Hoc Networks. Veh. Commun. 2018, 13, 64-77. [CrossRef]

35. Harrabi, S.; Jaffar, I.B.; Ghedira, K. Novel Optimized Routing Scheme for VANETs. Procedia Comput. Sci. 2016, 98, 32-39. [CrossRef]

36. Silva, A.; Reza, N.; Oliveira, A. Improvement and Performance Evaluation of GPSR-Based Routing Techniques for Vehicular Ad Hoc Networks. IEEE Access 2019, 7, 21722-21733. [CrossRef]

37. Almasri, A.; Chaddoud, G. Security of the Distributed Vehicular Broadcast Protocol DV-CAST. Int. J. Comput. Appl. 2020, 177, 26-31. [CrossRef]

38. Barba, T.C.; Colado, Z.A.; Aguiar, U.L.; Aguilar-Calderón, A.J. Survey on Routing Protocols for Vehicular Ad Hoc Networks Based on Multimetrics. Electronics 2019, 8, 1177. [CrossRef]

39. Bengag, A.; El Boukhari, M. Classification and comparison of routing protocols in VANETs. In Proceedings of the International Conference on Intelligent Systems and Computer Vision (ISCV), Fez, Morocco, 2-4 April 2018; pp. 1-8. [CrossRef]

40. Mathuranathan. Free Space Propagation Model. 2013. Available online: https://www.Gaussianwaves.Com/2013/09/Friss-FreeSpace-Propagation-Model/ (accessed on 22 November 2021).

41. Martinez, J.F.; Toh, K.C.; Cano, C.J.; Calafate, T.C.; Manzoni, P. Realistic Radio Propagation Models (RPMs) for VANET Simulations. In Proceedings of the IEEE Wireless Communications and Networking Conference, Budapest, Hungary, 5-8 April 2009; Volume 5, pp. 1-6.

42. Rappaport, S.T. Wireless Communications, Principles and Practice; Prentice Hall: Hoboken, NJ, USA, 1996.

43. Chandra, R. Performance Evaluation of Radio Propagation Model for Vehicular Ad Hoc Networks Using VanetMobiSim and NS-2. Int. J. Parallel. Emergent Distrib. Syst. 2012, 3, 145-155. 
44. Marchang, J.; Ghita, B.; Lancaster, D. Location Based Transmission Using a Neighbour Aware with Optimized EIFS MAC for Ad Hoc Networks. Ad Hoc Netw. 2017, 63, 62-78. [CrossRef]

45. Nakagami, M. The m-distribution-A general formula of intensity distribution of rapid fading. In Statistical Methods in Radio Wave Propagation; Elsevier: Pergamon, Turkey, 1960; pp. 3-36. 